\title{
Dual-Label STED Nanoscopy of Living Cells Using Photochromism
}

\author{
Katrin I. Willig,* Andre C. Stiel, Tanja Brakemann, Stefan Jakobs, and Stefan W. Hell* \\ Max-Planck-Institute for Biophysical Chemistry, Am Fassberg 11, 37077 Göttingen, Germany \\ Supporting Information
}

ABSTRACT: We demonstrate live-cell STED microscopy of two protein species using photochromic green fluorescent proteins as markers. The reversible photoswitching of two markers is implemented so that they can be discerned with a single excitation and STED wavelength and a single detection channel. Dual-label STED microscopy is shown in living mammalian cells.

KEYWORDS: Microscopy, two-color, superresolution, photoswitching, green fluorescent protein, RSFP

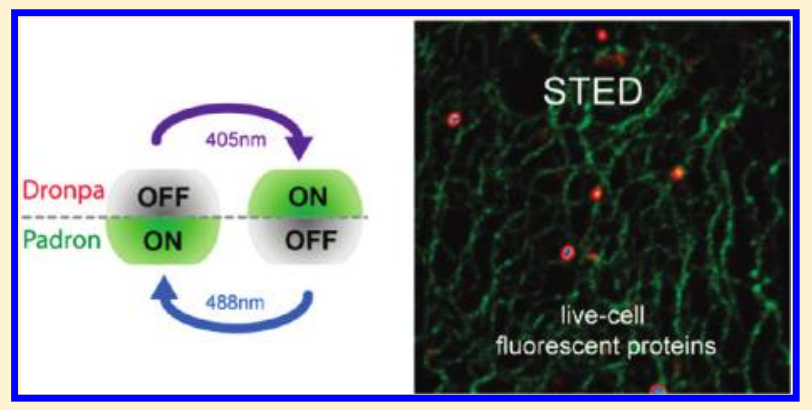

$\mathrm{E}$

mergent superresolution fluorescence microscopy (nanoscopy) displaying far-field optical resolution well beyond the diffraction limit has the potential to transform the life sciences. Over the last years several concepts for overcoming the diffraction barrier have been developed. While they all rely on modulating the fluorescence emission such that adjacent features fluoresce sequentially in time, these methods also boast differences in operation and strengths. ${ }^{1}$ Among the reported methods, STED fluorescence nanoscopy stands out by its ease of operation, wide range of applicability, recording speed, and ability to record $3 \mathrm{D}$ images from deep inside transparent specimens.

In a typical STED microscope, ${ }^{2,3}$ the majority of the fluorophores covered by the diffracted focal spot of excitation light are transiently rendered nonfluorescent, by preventing them from occupying the fluorescent state. This is accomplished by superimposing the spot of excitation light with a doughnut-shaped light spot of a wavelength inducing stimulated emission from the excited state of the fluorophore back down to its ground state. Only those fluorophores that happen to reside in subdiffraction proximity to the doughnut center are allowed to fluoresce. Scanning the beams across or through the sample forces adjacent features to fluoresce consecutively, so that they can be discerned by the detector.

Single-color live-cell STED microscopy has been shown in a variety of applications including the imaging of labeled vesicles at video rate, ${ }^{4}$ of cultured mammalians cells expressing various fusion proteins labeled with organic dyes, ${ }^{5,6}$ of YFP-labeled neurons in living organotypic brain slices, ${ }^{7}$ and others. Clearly, many applications require the imaging of two different fluorescent proteins in living cells. However, while STED microscopy has been extended to multiple colors, ${ }^{8}$ implementations of twocolor STED entailed (a combination of) additional pairs of laser lines, ${ }^{8,9}$ or an additional detection channel, ${ }^{8}$ or the use of fluorophores with long Stokes shifts. ${ }^{10-12}$ The latter also requires an additional excitation wavelength. While STED microscopy has recently been extended to three colors by exploiting a multilifetime analysis, ${ }^{13}$ two-color measurements in living cells have still been hampered by the complexity involved.

In this study we introduce a technically simple approach for STED microscopy of two fluorophores. The method is demonstrated with photochromic fluorescent proteins, which are genetically encoded and hence inherently compatible with living cells. Photochromic fluorescent proteins or reversible switchable fluorescent proteins (RSFPs) are structurally similar to the green fluorescent protein (GFP) but may be reversibly photoswitched from a nonfluorescent (off) to a fluorescent (on) state and back by irradiation with light of different wavelengths, whereby the longer switching wavelength also leads to fluorescence emission. RSFPs exhibit either a negative switching mode whereby the light eliciting fluorescence induces the off-switching or a positive switching mode in which the on-switching is induced. ${ }^{14}$

Here we use both the positive switching RSFP Padron ${ }^{14}$ and the negative switching Dronpa-M159T, ${ }^{15}$ which is an improved and faster switching variant of Dronpa ${ }^{16}$ (Figure 1A). The fluorescence of both proteins is induced by optical irradiation at $488 \mathrm{~nm}$. Moreover, Padron and Dronpa-M159T feature a similar spectrum peaking at 515 and $511 \mathrm{~nm}$, respectively (Figure 1B). To optimize these RSFPs for the expression in mammalian cells, we introduced the point mutation E218G and altered their $\mathrm{N}$ - and C-termini. Hence, we refer to these RSFPs as Padron $^{\mathrm{v} 2.0}$ and Dronpa-M159T $\mathrm{T}^{\mathrm{v} 2.0}$, with v2.0 standing for "improved version 2 ". Since the fluorescence excitation and emission spectra of both proteins are very similar, one can expect the same excitation and STED wavelengths to work for both. Importantly, because they exhibit antipodal switching characteristics, one should be able to switch these RSFPs successively to the on-state so that they are sequentially recorded by STED nanoscopy. Since the two RSFPs are separated by sequential switching rather than wavelength, the recording of Padron ${ }^{\mathrm{v} 2.0}$ and Dronpa-M159T $\mathrm{T}^{\mathrm{v} 2.0}$ is inherently

Received: July 6, 2011

Revised: July 20, 2011

Published: July 26, 2011 
monochromatic. Chromatic distortions are excluded. Our approach requires just a single additional wavelength for RSFP switching, which for the proteins in use was at $405 \mathrm{~nm}$.

To demonstrate this approach, we expressed Padron ${ }^{\mathrm{v} 2.0}$ fused to the microtubule associated protein Map2 (Padron ${ }^{\text {v2.0 }}$-Map2) in cultured mammalian cells and then Dronpa-M159T ${ }^{\mathrm{v} 2.0}$ fused to connexin37, which is a key component of gap junctions
(Cx37-Dronpa-M159T $\mathrm{T}^{\mathrm{v} 2.0}$ ). The fusion proteins highlighted the microtubule cytoskeleton and the gap junctions, respectively (Figure 2). To image the distribution of Padron ${ }^{\mathrm{v} 2.0}$-Map2 in the diffraction-limited confocal mode, we irradiated just with $488 \mathrm{~nm}$ light, which kept the proteins in the on state and concomitantly induced fluorescence. In the case of Cx37-Dronpa-M159T ${ }^{\mathrm{v} 2.0}$, the cells were additionally irradiated with light of $405 \mathrm{~nm}$ to keep

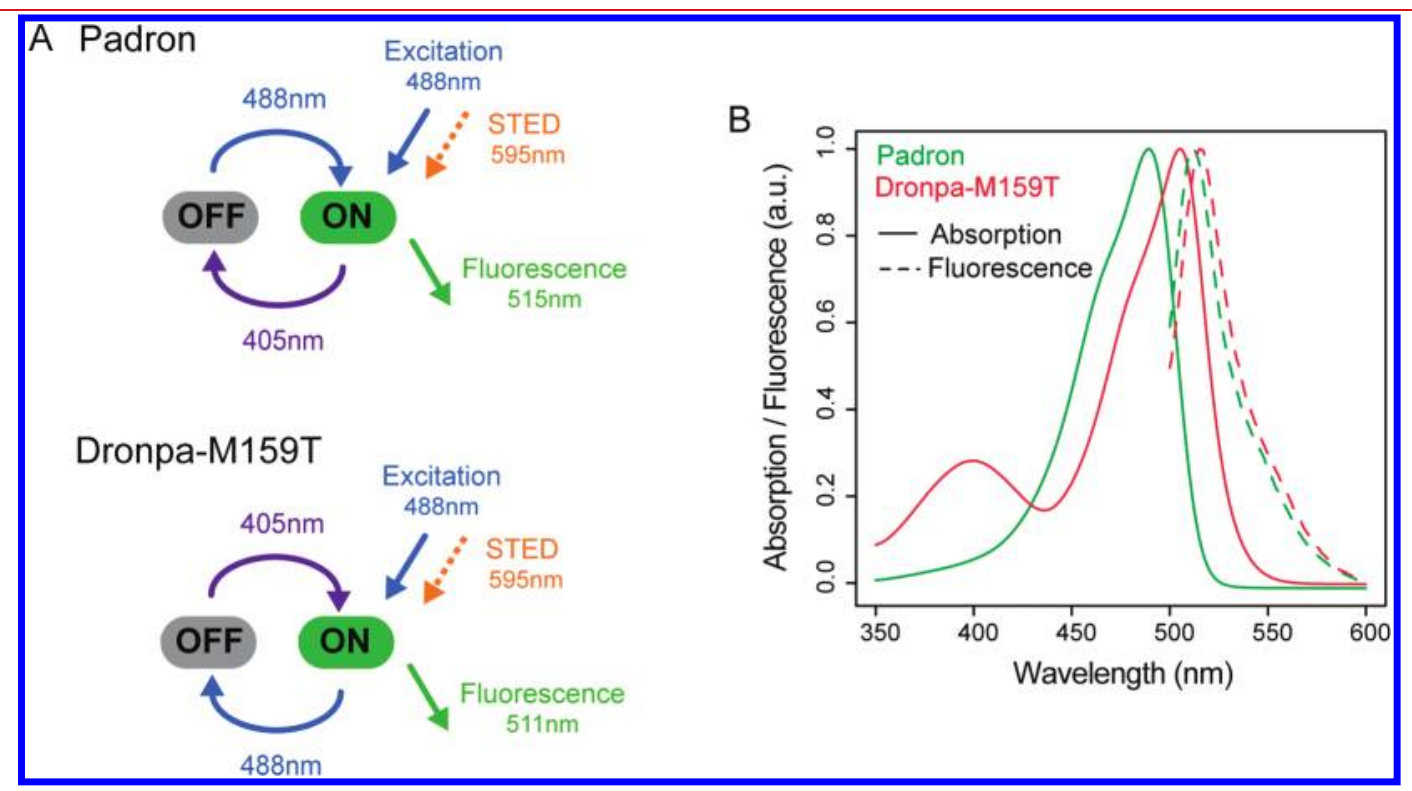

Figure 1. The photochromic fluorescent proteins Padron and Dronpa-M159T display antipodal switching behavior. (A) $488 \mathrm{~nm}$ light switches the fluorescence ability of Padron on and that of Dronpa-M159T off by transferring the proteins between two long-lived (on and off) states. $405 \mathrm{~nm}$ light reverses the switching: Padron is switched off and Dronpa-M159T on. In the on state, both proteins are excited with $488 \mathrm{~nm}$ light and fluorescence is transiently and instantly inhibited by stimulated emission (STED) at $595 \mathrm{~nm}$. (B) Absorption (solid line) and emission (dashed line) spectra recorded on purified protein solution for Padron (green) and Dronpa-M159T (red), both in the on-state.

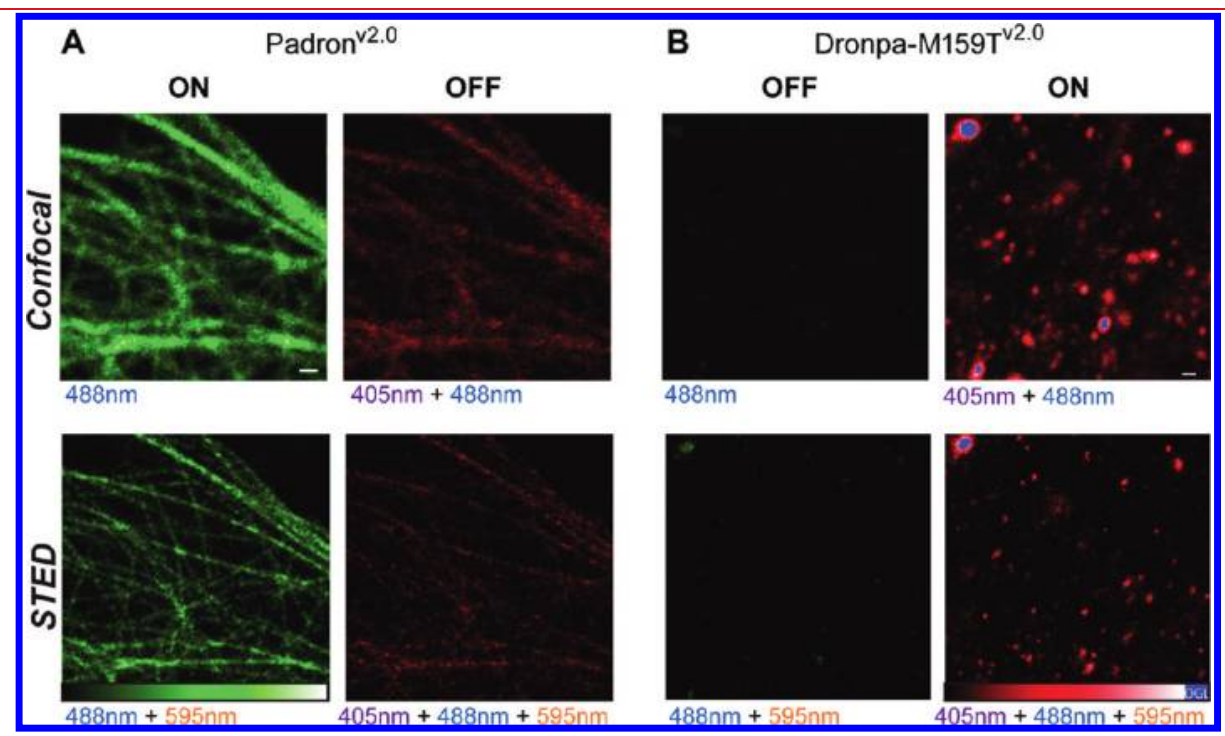

Figure 2. Cross talk of the Padron ${ }^{\mathrm{v} 2.0}$ and Dronpa-M159T ${ }^{\mathrm{v} 2.0}$ channel with and without STED. (A) Padron ${ }^{\mathrm{v} 2.0}$-Map2 highlights microtubules in PtK2 cell. It is switched on and read out with $488 \mathrm{~nm}$ light (green). Additional $595 \mathrm{~nm}$ STED light improves the resolution significantly beyond the diffraction limit. Switching off with additional $405 \mathrm{~nm}$ light yields a small signal which results in cross talk into the Dronpa-M159T ${ }^{\mathrm{v} 2.0}$ channel (red). (B) Cx37Dronpa-M159T $\mathrm{T}^{\mathrm{v} 2.0}$ forming gap junctions in the plasma membrane. No significant fluorescence is detected upon $488 \mathrm{~nm}$ illumination both with and without $595 \mathrm{~nm}$ STED light. Additional $405 \mathrm{~nm}$ light switches Dronpa-M159T $\mathrm{T}^{\mathrm{v} 2.0}$ to the on state showing clusters in the confocal mode and with subdiffraction resolution just by turning on the STED beam as well. The color maps of the "ON" and corresponding "OFF" images have the same range. Focal excitation power $1.4 \mu \mathrm{W}$ for all confocal and $4.2 \mu \mathrm{W}$ for all STED images; focal STED power $22.6 \mathrm{~mW}$. Scale bars $=500 \mathrm{~nm}$. 


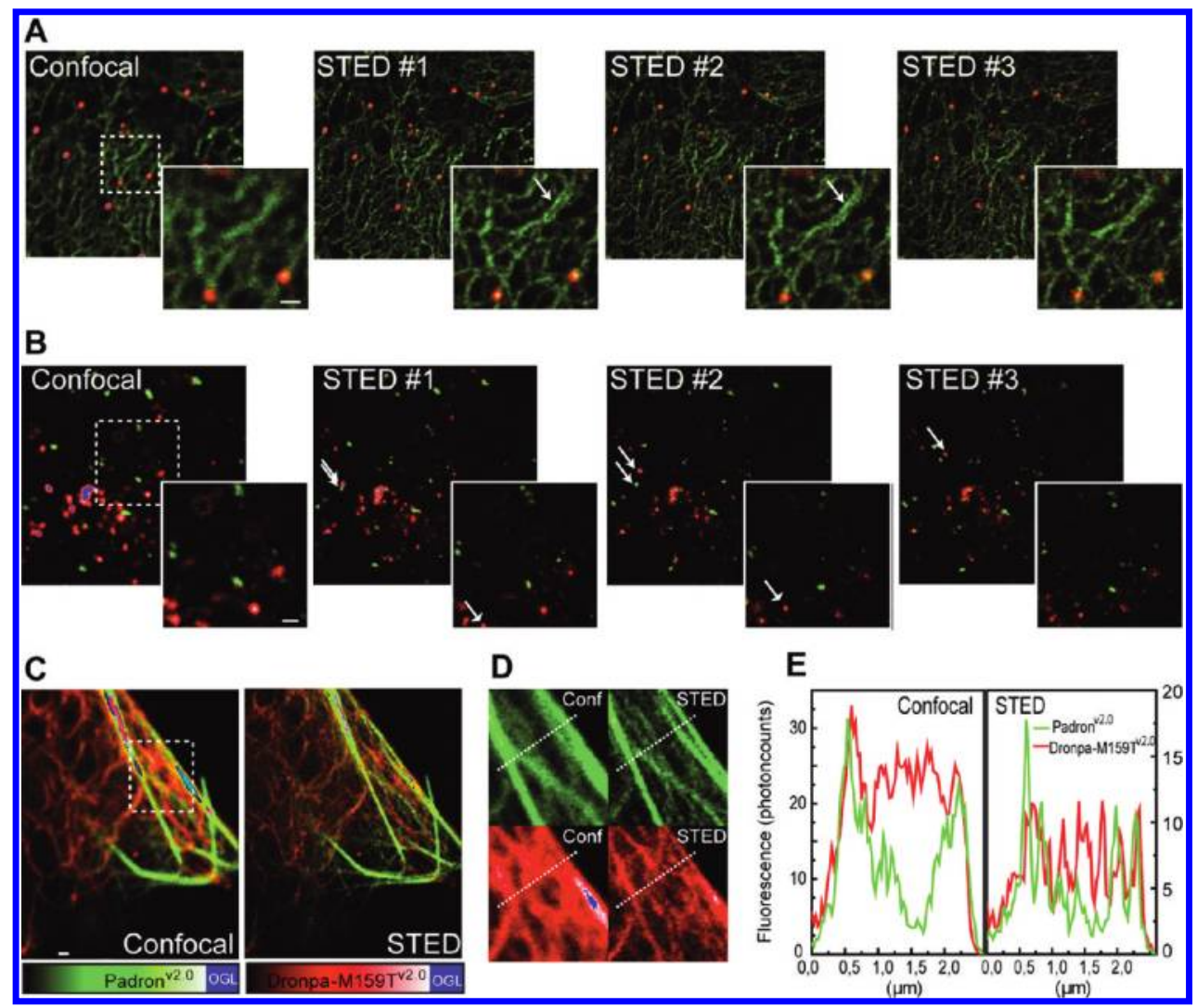

Figure 3. Live-cell STED microscopy using two reversible switchable fluorescent proteins. (A) The keratin intermediate filament network (green) of a PtK2 cell is marked with Krt19-Padron ${ }^{\mathrm{v} 2.0}$ and the peroxisomes (red) with Pex16-Dronpa-M159T ${ }^{\mathrm{v} 2.0}$. The STED image resolves the filaments of the keratin network which is blurred in the confocal image. Repeating the STED images (\#1, \#2,\#3) shows the dynamics of keratin network (highlighted by arrows) and peroxisomes in the living cells with subdiffraction resolution. (B) Gap junctions (Cx37-Padron ${ }^{\text {v2.0 }}$, green) and caveolin1 (Cav1-Dronpa-

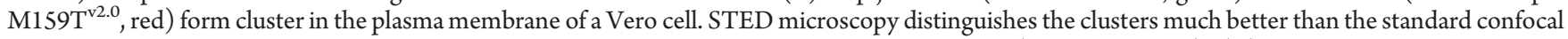
image and shows a small movement of the clusters between the STED images \#1, \#2, and \#3 (compare arrows). (C) Two cytoskeletal structures, Padron $^{\text {v2.0 }}$-Map2 (green) attached to microtubule and keratin (Krt19-Dronpa-M159T ${ }^{\text {v2.0 }}$, red) form an intricate mesh. STED microscopy reveals the network much better than the confocal mode. (D) Magnification of the boxed area in (C); display of the separate channels. (E) Line profiles averaged over three pixels of the dashed line in (D) show separately resolved fibers only by using STED microscopy. All images were recorded within $\sim 20 \mathrm{~s}$ per frame directly one after the other. The color maps of both channels of an image have the same range. Excitation and STED power equivalent to those used in Figure 2, besides excitation \#2 STED, $8.3 \mu \mathrm{W}$; \#3 STED, $11.4 \mu \mathrm{W}$. Scale bars $=500 \mathrm{~nm}$.

the proteins in the on state during imaging. Both Padron ${ }^{\mathrm{v} .0}$ and Dronpa-M159T $\mathrm{T}^{\mathrm{v} 2.0}$ could be imaged in the STED mode using light of $595 \mathrm{~nm}$ for stimulated emission (Figure 2).

The images demonstrate the resolution improvement gained by STED, as structures are clearly discernible in the STED recordings which are blurred in their confocal counterparts. The residual fluorescence of Dronpa-M159T ${ }^{\mathrm{v} 2.0}$ residing in the off state was below $2 \%$ of that of its on-state fluorescence in the confocal mode and 9\% in the STED mode, demonstrating the good switching contrast obtainable with this protein. With the same light intensities, Padron ${ }^{\mathrm{v} 2.0}$ was switched to $30 \%$ of the maximal fluorescence in both the confocal and the STED mode (Figure 2A). We note that in the case of Padron ${ }^{\mathrm{v} 2.0}$, the switching contrast can be further improved by adjusting the light intensities, if required. Together, these data demonstrate that $\mathrm{Pa}-$ dron $^{\text {v2.0 }}$ and Dronpa-M159T ${ }^{\mathrm{v} 2.0}$ can be expressed as functional fusion proteins in living cells, that both proteins facilitate subdiffraction imaging in the STED mode, and that they can be antagonistically photoswitched such that the two protein species can be discriminated.

To demonstrate STED imaging of living cells coexpressing Padron $^{\text {v2.0 }}$ and Dronpa-M159T $\mathrm{T}^{\mathrm{v} 2.0}$, we fused both RSFPs to different host proteins. Figure 3A shows the intermediate filament network (Krt19-Padron ${ }^{\text {v2.0 }}$, green) and the peroxisomes (Pex16-Dronpa-M159T $\mathrm{T}^{\mathrm{v} .0}$, red) imaged in the confocal and STED microscopy mode. STED microscopy resolves individual filaments within the network and the peroxisomes as small spherical structures. Repetitive STED imaging reveals slight dynamical changes in the structures (see arrows in Figure 3A). Next, we tagged two proteins which are known to form clusters in the plasma membrane: the gap junction protein connexin 37 $\left(\mathrm{Cx} 37-\right.$ Padron $^{\mathrm{v} 2.0}$ ) and caveolin1 (Cav1-Dronpa-M159T ${ }^{\mathrm{v} 2.0}$ ). STED resolves the clusters in much finer detail than its diffraction-limited confocal counterpart (Figure 3B). Again, several STED images could be recorded on the same cell, demonstrating some movements of the protein clusters within the plasma membrane. Finally, we colabeled the microtubule and the intermediate filament cytoskeleton with Padron ${ }^{\text {22.0 }}$-Map2 and Krt19Dronpa-M159T $^{\mathrm{v} 2.0}$ (Figure 3C). These dense networks are highly interwoven in PtK2 cells. Whereas they appear largely blurred in the confocal image, individual filaments can be clearly separated in the STED images (Figure 3C-E).

In STED microscopy, the attainable resolution depends on the applied STED laser intensity and the cross section for stimulated 
emission. We adjusted the STED intensity so that several consecutive images in both channels could be recorded. A higher STED power would have resulted in a higher resolution at the expense of increased photobleaching. Under the chosen experimental conditions, we found that in the dual-label images, the full width at half-maximum (fwhm) of small structures (which is an estimate of the resolution) was $\sim 70 \mathrm{~nm}$ or $\sim 90 \mathrm{~nm}$ in the case of Padron $^{\mathrm{v} 2.0}$ or Dronpa-M159T ${ }^{\mathrm{v} 2.0}$-labeled structures, respectively. Presumably, the slightly lower resolution in the DronpaM159T ${ }^{\mathrm{v} 2.0}$ STED images can be attributed to two properties of this fluorophore: (i) its fluorescence emission is blue-shifted by $4 \mathrm{~nm}$ as compared to Padron ${ }^{\mathrm{v} 2.0}$, lowering the cross section for stimulated emission, (ii) the fluorescence lifetime of Dronpa$\mathrm{M}_{159 \mathrm{~T}^{\mathrm{v} 2.0}}$ is only $0.8 \mathrm{~ns}$ which is much shorter than the $2.5 \mathrm{~ns}$ fluorescence lifetime of EYFP which has previously been used in STED microscopy. Also, it is much shorter than the $3.0 \mathrm{~ns}$ lifetime of Padron ${ }^{\mathrm{v} .0}$. All these factors reduce the STED efficiency further, making STED of Dronpa-M159T $\mathrm{T}^{\mathrm{v} 2.0}$ more sensitive to pulse timing jitter. ${ }^{17}$

To minimize the spurious autofluorescence induced by the $405 \mathrm{~nm}$ line, we also implemented a modality in which we alternated the irradiation with the 488 and $405 \mathrm{~nm}$ laser lines. Whereas in the first half of the resonant scanner duty cycle one of the lines was on, in the second half the other line was on, while the signal of both duty cycles was collected separately to form an image. However, this measure is not strictly needed because the autofluorescence background can also be avoided by spatially offsetting the beams. The $405 \mathrm{~nm}$ switching light spot can be slightly displaced in which case the autofluorescence is not sensed by the confocal detection. Confocal detection was used because it conveniently suppresses the background. Note that neither STED microscopy per se nor this dual-label version strictly require the use of a confocal arrangement.

Importantly, this study has been performed with two photochromic proteins that were not specifically optimized for STED microscopy. Hence future generations of RSFPs specifically tailored for this approach or smart labels using photochomic synthetic fluorophores are likely to facilitate higher imaging speeds as well as higher resolution values. In summary, the use of photochromic fluorescent proteins enabled the first dual-label STED microscopy recording in living cells. Using a single wavelength channel for excitation, STED, and detection, the concept is readily implemented and hence should find wide use in superresolution imaging of living cells.

\section{ASSOCIATED CONTENT}

S Supporting Information. A Materials and Methods section describing the new termini, the plasmid generation, cell preparation, and STED setup. This material is available free of charge via the Internet at http://pubs.acs.org.

\section{AUTHOR INFORMATION}

\section{Corresponding Authors}

*E-mail: shell@gwdg.de (S.W.H.); kwillig@gwdg.de (K.I.W.).

\section{ACKNOWLEDGMENT}

We thank Tanja Gilat, Sylvia Löbermann, and Ellen Rothermel for cell culturing and plasmid generation, Birka Lalkens for creative and supportive help with the double labeling, Volker
Westphal and Andreas Schönle for help with the scanning hardand software, and Jaydev Jethwa for critical reading of the manuscript. Parts of this work were supported by Deutsche Forschungsgemeinschaft through the DFG-Research Center for Molecular Physiology of the Brain (to S.J.) and the Leibniz prize of the Deutsche Forschungsgemeinschaft (to S.W.H.).

\section{REFERENCES}

(1) Hell, S. W. Microscopy and its focal switch. Nat. Methods 2009, 6 (1), 24-32.

(2) Hell, S. W.; Wichmann, J. Breaking the diffraction resolution limit by stimulated-emission-stimulated-emission-depletion fluorescence microscopy. Opt. Lett. 1994, 19 (11), 780-782.

(3) Klar, T. A.; Jakobs, S.; Dyba, M.; Egner, A.; Hell, S. W. Fluorescence microscopy with diffraction resolution barrier broken by stimulated emission. Proc. Natl. Acad. Sci. U.S.A. 2000, 97, 8206-8210.

(4) Westphal, V.; Rizzoli, S. O.; Lauterbach, M. A.; Kamin, D.; Jahn, R.; Hell, S. W. Video-Rate Far-Field Optical Nanoscopy Dissects Synaptic Vesicle Movement. Science 2008, 320 (5873), 246-249.

(5) Hein, B.; Willig, K. I.; Wurm, C. A.; Westphal, V.; Jakobs, S.; Hell, S. W. Stimulated Emission Depletion Nanoscopy of Living Cells Using SNAP-Tag Fusion Proteins. Biophys. J. 2010, 98, 158-163.

(6) Fitzpatrick, J. A. J.; Yan, Q.; Sieber, J. J.; Dyba, M.; Schwarz, U.; Szent-Gyorgyi, C.; Woolford, C. A.; Berget, P. B.; Waggoner, A. S.; Bruchez, M. P. STED Nanoscopy in Living Cells Using Fluorogen Activating Proteins. Bioconjugate Chem. 2009, 20 (10), 1843-1847.

(7) Nägerl, U. V.; Willig, K. I.; Hein, B.; Hell, S. W.; Bonhoeffer, T. Live-cell imaging of dendritic spines by STED microscopy. Proc. Natl. Acad. Sci. U.S.A. 2008, 105 (48), 18982-18987.

(8) Donnert, G.; Keller, J.; Wurm, C. A.; Rizzoli, S. O.; Westphal, V.; Schoenle, A.; Jahn, R.; Jakobs, S.; Eggeling, C.; Hell, S. W. Two-Color Far-Field Fluorescence Nanoscopy. Biophys. J. 2007, 92 (8), L67-L69.

(9) Neumann, D.; Bückers, J.; Kastrup, L.; Hell, S.; Jakobs, S. Twocolor STED microscopy reveals different degrees of colocalization between hexokinase-I and the three human VDAC isoforms. PMC Biophys. 2010, 5 (3), 1-4.

(10) Schmidt, R.; Wurm, C. A.; Jakobs, S.; Engelhardt, J.; Egner, A.; Hell, S. W. Spherical nanosized focal spot unravels the interior of cells. Nat. Methods 2008, 5 (6), 539-544.

(11) Gugel, H.; Giske, A.; Dyba, M.; Sieber, J., Confocal imaging at the nanoscale with two-color STED microscopy. Proc. SPIE 2011, 7905.

(12) Friedemann, K.; Turshatov, A.; Landfester, K.; Crespy, D. Characterization via Two-Color STED Microscopy of Nanostructured Materials Synthesized by Colloid Electrospinning. Langmuir 2011, 27 (11), 7132-7139.

(13) Bückers, J.; Wildanger, D.; Vicidomini, G.; Kastrup, L.; Hell, S. W. Simultaneous multi-lifetime multi-color STED imaging for colocalization analyses. Opt. Express 2011, 19 (4), 3130-3143.

(14) Andresen, M.; Stiel, A. C.; Folling, J.; Wenzel, D.; Schonle, A.; Egner, A.; Eggeling, C.; Hell, S. W.; Jakobs, S. Photoswitchable fluorescent proteins enable monochromatic multilabel imaging and dual color fluorescence nanoscopy. Nat. Biotechnol. 2008, 26 (9), 1035-1040.

(15) Stiel, A. C.; Trowitzsch, S.; Weber, G.; Andresen, M.; Eggeling, C.; Hell, S. W.; Jakobs, S.; Wahl, M. C. 1.8 angstrom bright-state structure of the reversibly switchable fluorescent protein dronpa guides the generation of fast switching variants. Biochem. J. 2007, 402 (1), $35-42$.

(16) Ando, R.; Mizuno, H.; Miyawaki, A. Regulated Fast Nucleocytoplasmic Shuttling Observed by Reversible Protein Highlighting. Science 2004, 306 (5700), 1370-1373.

(17) Leutenegger, M.; Eggeling, C.; Hell, S. W. Analytical description of STED microscopy performance. Opt. Express 2010, 18 (25), 26417-26429. 